\title{
Does arch length preservation in mixed dentition children affect mandibular second permanent molar eruption? A systematic review and meta-analysis
}

Sivakumar Arunachalam ${ }^{1 *} \mathbb{D}$, Indumathi Sivakumar ${ }^{2}$, Jayakumar Jayaraman ${ }^{3}$ and Jitendra Sharan ${ }^{4}$

\begin{abstract}
Background: Arch length preservation strategies utilize leeway space or E-space in the mixed dentition to resolve mild to moderate mandibular incisor crowding. The purpose of this systematic review of the literature was to analyze the effects of arch length preservation strategies in on mandibular second permanent molar eruption.

Methods: A search for relevant articles published from inception until May 2020 was performed using PubMed/ Medline, Cochrane databases, Clinicaltrials.gov, Google scholar and journal databases. Preferred Reporting Items for Systematic Reviews and Meta-Analyses (PRISMA) guidelines were adopted for the conduct of the systematic review. Using RevMan 5.3 software, the most pertinent data were extracted and pooled for quantitative analysis with 95\% confidence intervals. Heterogeneity was analyzed by using Cochran Q test and I squared statistics.

Results: A total of 5 studies involving 855 mixed dentition patients with arch length preservation therapy were included in the qualitative analysis. Pooled estimate of the data from two studies revealed 3.14 times higher odds of developing mandibular second molar eruption difficulty due to arch length preservation strategies using lingual holding arch (95\% Cl; OR 1.10-8.92). There was no heterogeneity found in the analysis. The certainty levels were graded as very low.
\end{abstract}

Conclusions: This systematic review demonstrates that arch length preservation strategies pose a risk for development of mandibular second molar eruption disturbances, but the evidence was of very low quality.

Registration number: CRD42019116643.

Keywords: Leeway space, E- space, Arch length, Lip bumper, Lingual holding arch, Molar impaction, Eruption difficulties

\footnotetext{
*Correspondence: sivakumar@imu.edu.my

${ }^{1}$ School of Dentistry, International Medical University, No.126, Jalan Jalil

Perkasa 19, Bukit Jalil, Kuala Lumpur, Malaysia

Full list of author information is available at the end of the article
}

\begin{abstract}
Background
Loss of mandibular arch length is an inevitable event during the transitional period, and it was estimated to be about $1.8 \mathrm{~mm}$ per side of the arch [1]. This phenomenon raised a reasonable question that if simple arch length preservation during the transition period could provide adequate space to manage crowding in the mixed dentition without any active intervention [2]. Accordingly, utilization of leeway space of
\end{abstract}


Nance/E-space just prior to exfoliation of the mandibular second primary molar through arch length preservation strategies (ALPS) for the relief of mandibular anterior crowding has been suggested [2-4].

Several investigators consistently demonstrated the effectiveness of arch length preservation in preventing mesial migration of the permanent first molars [5-7]. A recent systematic review reported $5.1 \mathrm{~mm}$ resolution of mandibular incisor crowding with passive lower lingual arch therapy [8]. However, conflicting notion exists in the literature with regards to the early management of mandibular incisor crowding and resultant long-term dental health benefits $[9,10]$. Further, clinical studies could not demonstrate long-term lower incisor positional stability through ALPS when compared to mixed dentition expansion protocols or extraction of premolars [11].

With the probability of successful early management of crowding using arch length preservation strategies, researchers attempted to explore further on the process of natural transitional mechanism in the dentition and its impediments, if any $[12,13]$. They noted that it may not be prudent to manage the anterior arch discrepancy without creating a posterior arch discrepancy [12]. On a general note, Paulo and Betty demonstrated some risk of mandibular second permanent molar (M2) impaction in a sample of patients undergoing orthodontic treatment [14].

More recently, studies utilizing ALPS have reported an increase in the incidence of M2 eruption difficulties leading to impaction or ectopic eruption [15-19]. A reported incidence in the range of $4.7-14.5 \%$ was noted with lingual holding arch and $11.9-22 \%$ with lip bumper [15-19]. However, prevalence of impacted $\mathrm{M} 2 \mathrm{~s}$ in the general population ranged from 0.2 to $2.3 \%$ $[20,21]$. The aim of this study was to systematically review the effects of ALPS in mixed dentition on mandibular second permanent molar eruption.

\section{Methods}

\section{Protocol and registration}

Guidelines from 'Preferred Reporting Items for Systematic Reviews and Meta-Analyses (PRISMA)' helped to report this review in concordance [22]. The review protocol was registered in PROSPERO International Prospective Register of Systematic Reviews (CRD42019116643).

\section{Eligibility criteria}

The methodology included formulating review questions using a Population, Exposure, Comparison, Outcome, Study design (PECOS) framework (Table 1), constructing a search strategy, defining inclusion and exclusion criteria, locating studies, selecting studies, assessing study quality, extracting data, and forming an evidence table prior to interpretation. The research question formulated for this study was as follows: Does arch length preservation strategies in the mixed dentition affect mandibular second permanent molar eruption?

This review considered the studies pertaining to arch length preservation utilizing lingual holding arch and lip bumper appliance as an interceptive procedure (nonextraction treatment). The study designs included randomized controlled trials (RCTs), prospective cohort studies, and retrospective studies. All the studies should have reported follow-ups before and after orthodontic evaluation. The review included all publications from different languages without any restriction. Exclusion included scripts from review papers, letters to editor, case reports, cases with extraction modality, multiple publications on same pool of patients, and animal studies on the review topic.

\section{Information sources and search}

An electronic search was conducted in the following databases to identify the relevant studies: National library of Medicine (MEDLINE-PubMed) via PubMed, Cochrane Central Register of Controlled Trials, Cochrane's Oral Health Group's Trials Register, Clinicaltrials.gov, Google scholar, and other journal databases (Elsevier, Wiley, Oxford Academic, SAGE journals) from inception up to September 2020. A manual search of the reference source from all the selected full text articles and review articles on the subject identified relevant studies. Table 2 tabulates search strategy and key words.

Table 1 Population, exposure, comparison, outcome, study design (PECOS) framework

\begin{tabular}{ll}
\hline Population & Children with mixed dentition \\
\hline Exposure & Orthodontic treatment with arch length preservation strategies in the mandibular arch \\
Comparison & Untreated control group of children, children with treatment other than arch length preservation \\
Outcomes & Mandibular second permanent molar eruption disturbances as evidenced radiographically \\
Study design & Randomized control trials \\
& Prospective cohort studies \\
& Retrospective studies \\
\hline
\end{tabular}




\section{Study selection}

Two independent reviewers (SA and IS) scrutinized titles and abstracts of the potentially qualifying studies. The reviewers conducted the assessment of the full texts independently for relevance. A third reviewer (JJ) resolved any disagreement between the first two reviewers by consensus.

\section{Data collection process and data items}

Data were extracted by 2 reviewers independently from each included study and entered in an electronic spreadsheet that included the following information: name of the author, year of publication, study design, sample size, inclusion criteria, appliance type (arch length preservation strategy), appliance wear duration, treatment duration, outcome assessment, mean anterior mandibular crowding, M2 eruption problems, and percentage of eruption difficulty.

\section{Risk of bias (ROB) within studies}

The quality assessment tool for observational cohort and cross-sectional studies assessed the selected studies independently [23]. This assessment tool contained 14 questions focusing on the assessment of the internal validity of the study. Each study was evaluated based on the information of the study design and execution and how well the confounding factors were handled to minimize bias. Accordingly, the tool accorded good, fair, or poor ratings to the studies. The Kappa $(\mathrm{k})$ coefficient formalized the agreement between the reviewers with data extraction [24].

\section{Summary measures}

Measurements for the outcome were based on nominal data which provides information about impaction or eruption difficulty from dental radiographs.

\section{Synthesis of results}

Guidance from the Cochrane handbook of systematic review and RevMan 5.3 software (Review Manager, RevMan V.5.3, Copenhagen, The Nordic Cochrane Centre, The Cochrane Collaboration, 2014) assisted to perform the meta-analysis in a fixed-effects model [25]. Metaanalysis was performed for two studies with controls that employed lingual holding arch as a means of arch length preservation. The dichotomous data were presented as odds ratio and 95\% confidence interval (CI). Cochran $\mathrm{Q}$ test along with I squared statistics estimated the heterogeneity. I squared statistics range from 0 to $100 \%$. An I squared index less than $25 \%$ is indicative of low heterogeneity, between $75 \%-25 \%$ represents average

Table 2 Search strategy

Database PubMed

Cochrane registry, CENTRAL Clinicaltrails.gov, Google scholar, journal database (Elsevier, Wiley, Oxford Academic, SAGE journals)

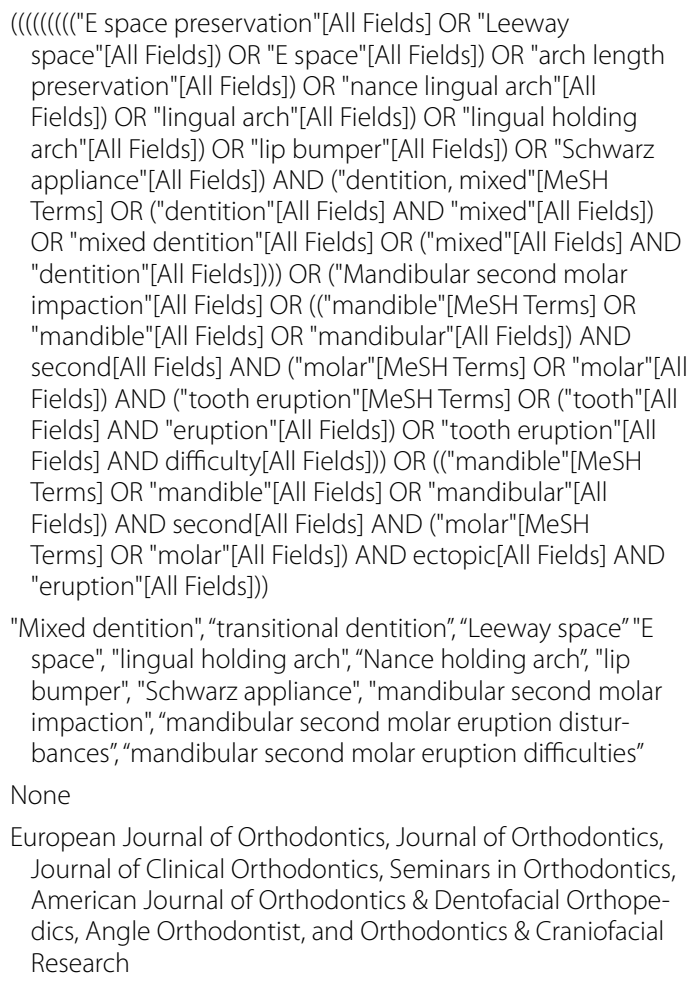

"Mixed dentition", "transitional dentition", "Leeway space" "E space", "lingual holding arch","Nance holding arch", "lip bumper", "Schwarz appliance", "mandibular second molar impaction","mandibular second molar eruption disturbances", "mandibular second molar eruption difficulties" None

European Journal of Orthodontics, Journal of Orthodontics, Journal of Clinical Orthodontics, Seminars in Orthodontics, American Journal of Orthodontics \& Dentofacial Orthopedics, Angle Orthodontist, and Orthodontics \& Craniofacial Research 
heterogeneity, and more than $75 \%$ means that considerable heterogeneity is present [26].

\section{Risk of bias across studies}

The quality of evidence of the outcome in the meta-analysis was evaluated using the Grading of Recommendations Assessment, Development, and Evaluation (GRADE) system. The following criteria were included for assessment of the quality of evidence for the outcome across studies: study design; ROB; consistency; precision; publication bias; and other considerations. Consistency was judged based on the heterogeneity (I squared) of the outcome and was ranked as: not serious-zero to $30 \%$; serious -30 to $75 \%$; and very serious-greater than 75 percent. Precision was judged based on the crossing of the CI of the pooled outcome to the no-effect line and the total sample size; it was ranked as "not serious" if total sample size was larger than 40, "serious" if between 20 and 40, and "very serious" if smaller than 20. Publication bias could be assessed when outcome had more than 10 articles included for quantitative analysis. The GRADE system results in four grades in rating the quality of evidence: (1) high; (2) moderate; (3) low; and (4) very low.

\section{Results}

\section{Study selection}

200 studies were obtained from electronic search and two records were identified through hand searching. After duplicates were removed, 185 records remained. Another 173 records were excluded after reading the titles and abstracts. A total of twelve records were found eligible for full text screening. Following full-text assessments, 7 articles were excluded: 6 articles were narrative or systematic reviews related to the use of similar appliances (lingual holding arch/lip bumper) but with different outcome measurements, and 1 article was a conference abstract on the topic. Finally, five studies were included in qualitative synthesis [15-19] and two studies were included for meta-analysis [17, 18] (Fig. 1). The Kappa statistic indicated "almost perfect" inter- examiner reviewer agreement ( $k=0.91,95 \%$ CI: 0.89 to 0.94$)$.

\section{Study characteristics}

Detailed descriptive data of the included studies are listed in Table 3. Of the five included studies, two were prospective cohort studies $[16,17]$ and the remaining three were retrospective studies $[15,18,19]$. Two studies used a lip bumper $[15,19]$ and three studies used a lingual holding arch [16-18]. The search did not identify any randomized controlled trials. The year of publication of the included studies ranged from 2011 to 2020. In total, 1222 participants were part of these studies, out of which 855 participants underwent arch length preservation for the relief of minor crowding and 367 participants were in the control group. Of the two lip bumper studies, only one reported full time wear (24 h per day) [15] and the other did not specify [19]. Also, it was reasonable to assume that the lingual holding arch is not removable and therefore would be worn full time [16-18]. The treatment duration ranged from 7 to 75 months.

\section{Risk of bias within studies}

Quality assessment of the included studies revealed that one study was good, three studies were fair, and another study was poor with moderate risk of bias (Table 4). Only two studies reported sample size calculation $[17,18]$, and this could implicate the lack of adequate size and effect in other studies. Statistical analysis to control the potential confounding variables which were not of interest were measured in four of the included studies [15-18].

\section{Results of individual studies}

The mechanism for arch length preservation varied between the strategies including harnessing the force from the lip during normal oral functions as in lip bumper or maintaining a passive support with lingual holding arch. Mandibular second molar eruption problems were noticed in lip bumper that ranged from 11.9 to $22 \%$ and passive lingual holding arch that varied between 4.7 and $14.5 \%$ [15-19]. These were primarily based on the status of eruption and the stage of root development, or position of the mesial cusps below the height of contour of the distal surface of the mandibular first molar (Table 5). However, one study failed to clearly describe the criteria for measuring the study outcome [19]. Of the 855 subjects, 130 subjects experienced M2 eruption problems with the difficulty of eruption ranging from 11.9 to $22 \%$. The control group as reported by three studies, that demonstrated a prevalence of M2 eruption problems ranged from 1 to $2.96 \%$ [15, 17, 18]. One study did not provide details about the historic controls and hence, the control group details could not be considered in the analysis [16]. The nature of eruption difficulty included either impaction or ectopic eruption. A common predictor for the development of M2 impaction was the angulation greater than 24 degrees between first molar and M2 angulation concomitant with arch length preservation strategies $[15,16]$. However, one study reported that a greater angulation could not be considered a significant predictor of M2 eruption difficulty [17]. With lip bumper protocol, distal tipping of the first molars or incorrect fitting of the first molar bands have been noted as the possible causes for M2 impaction [15, 19]. With lingual holding arch, either space-width ratio or molar angulation has been implicated as a predictor for M2 impaction $[16,17]$. 


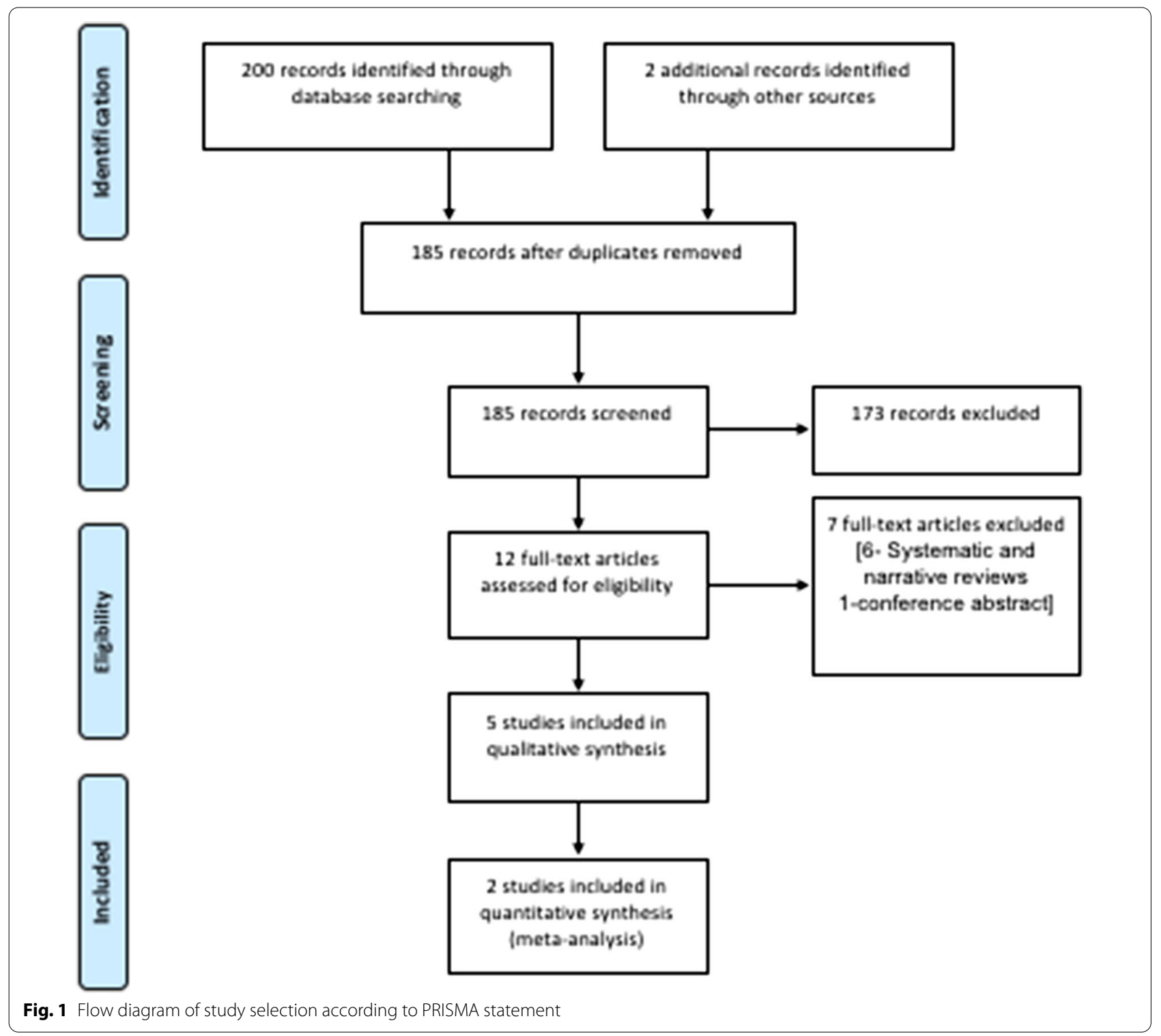

\section{Synthesis of results}

Meta-analysis included two studies that employed lingual arch, and that reported M2 eruption difficulty ranging from 4.7 to $7.1 \%$ [17, 18]. The pooled data from the two studies revealed 3.14 times higher odds of developing M2 eruption difficulty at 95\% CI (1.10-8.92). The studies observed no heterogeneity in the analysis (Fig. 2). Since there are only two studies contributing to the data for meta-analysis, we were unable to present the sensitivity analysis by excluding the studies. However, in addition to the analysis presented, we included a pooled estimation of intervention group percentage of difficulties in eruption grouping all studies using $\mathrm{R}$ software (Fig. 3). The aggregate eruption difficulty is around $12 \%$ based on five studies.

\section{Risk of bias across studies}

The certainty of evidence was evaluated according to the GRADE approach. The bias elements were not downgraded in the grade approach. However, there is a serious problem: the imprecision domain in grade where the $95 \% \mathrm{CI}$ is too wide to arrive at a precise conclusion. Funnel plots were not constructed as the data available for the meta-analysis is only two studies. For the outcome, mandibular second molar eruption disturbances, the certainty levels were graded as very low (Table 6). 


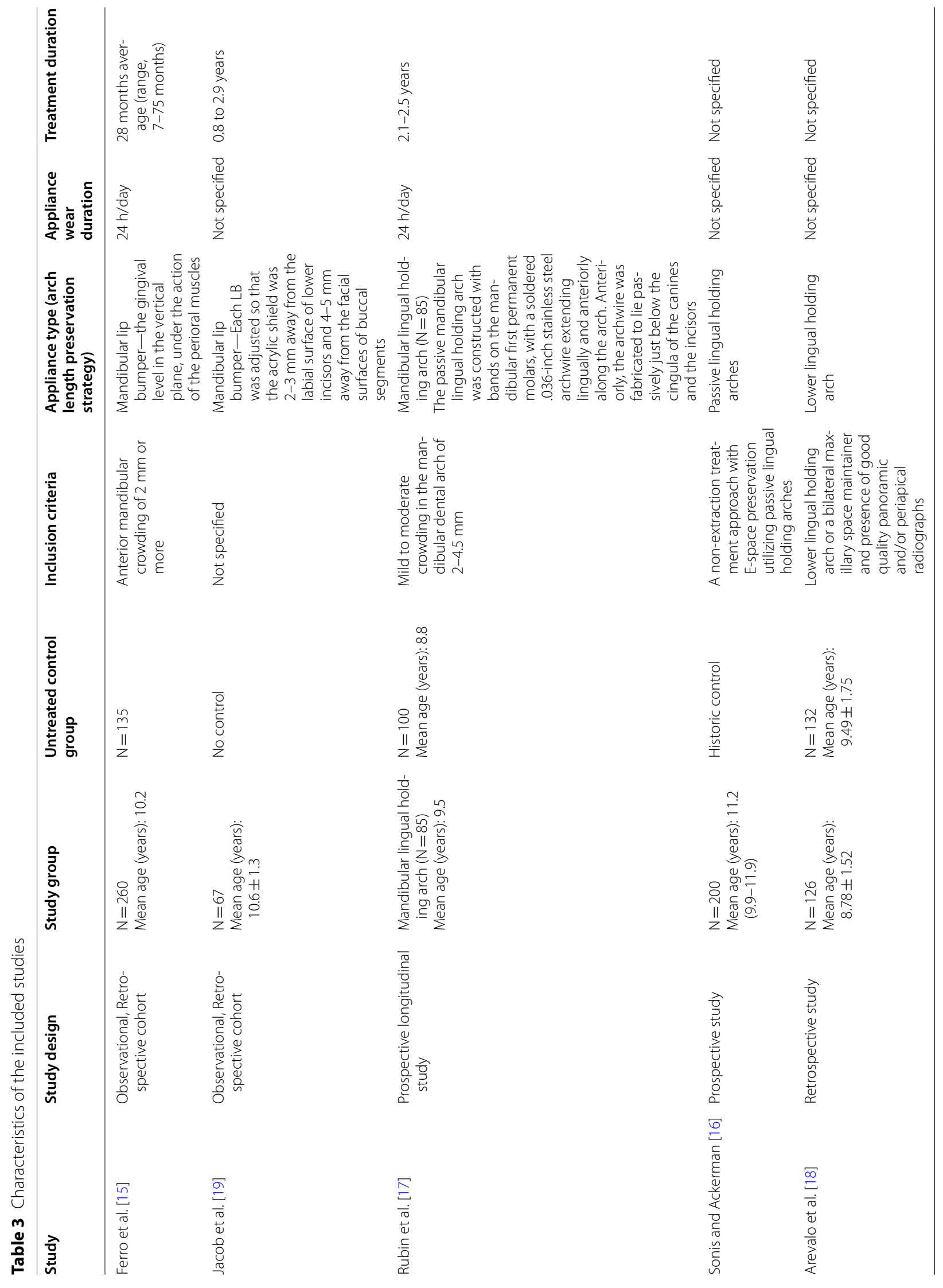




\section{Discussion}

\section{Summary of evidence}

This systematic review and meta-analysis explored and summarized the information associated with ALPS and potential M2 eruption difficulties, both ectopic eruption and impaction. There are two reviews on ALPS: one was a narrative review that studied the use of lip bumper and its subsequent effect on M2 eruption but included only case reports and other narrative reviews [27]. This narrative review reported $\mathrm{M} 2$ impaction in $7-12 \%$ of the treated group and $1.4 \%$ in the untreated group. The second was a systematic review that evaluated the effects of lip bumper therapy on the mandibular dental arch of children and adolescents as the primary outcome and M2 eruption disturbances as the secondary outcome [28]. A number of other studies reported first molar distalization or arch length changes with lip bumpers when compared with untreated controls [29-33]. A recent review reported that lingual arch did not increase the arch length significantly negating any change in the position of the mandibular first molars [8]. However, Viglianisi in another systematic review demonstrated $0.54^{\circ}$ of first molar distal tipping with lingual arch [3]. Hence it is understandable, that the effect on the M2 eruption with lingual arch will be minimal. To avoid a possible bias, only lingual holding arch strategy was considered in the quantitative analysis. Till date, there is no systematic review or meta-analysis addressing lingual holding arch effects on the M2 eruption. Current meta-analysis revealed 3.14 times higher odds of developing mandibular M2 eruption difficulty with 95\% CI (1.10-8.92) after arch length preservation modality with lingual holding arch. However, it is to be noted that the outcomes were pooled data of retrospective studies $[15,18,19]$ and prospective cohort studies $[16,17]$.

The mandibular second molar eruption problems were noticed with both the active and passive strategies. In the active mode (lip bumper), it ranged from 11.9 to $22 \%$ and passive mode (lingual holding arch) demonstrated eruption problems ranged between 4.7 and $14.5 \%$. The pooled estimate of eruption difficulties in the intervention group demonstrated a $12 \%$. Given the influence of active nature of the appliance on the first molar, the lip bumpers not only maintained arch length, thus preserving leeway space, but (particularly if advanced) distalisation of the lower first molars took place [29, 34]. However, few investigations noted that the changes in the arch length happened irrespective of the second molar status [35, 36]. There are equivocal conclusions with regards to predictive factors for mandibular M2 in the literature [15-17]. For lingual arch therapy, Sonis and Ackerman reported an increased risk of M2 impaction when the inter-molar angulation exceeded $24^{\circ}$ [16], but contradictory to Rubin et al. assessment where higher angulation is not a predictor [17]. First molar/M2 spacing, presence of third molar, space width ratio, facial pattern, skeletal relationship, gender, and age proved to be poor predictors of mandibular M2 eruption difficulty [14-17]. Arevalo et al. on lingual arch ALPS noted that there was 6.53 times greater chance of M2 impaction compared to controls after controlling for age [18]. For every increase in age by one year, there was an increase in the odds of M2 impaction by 1.25 times after controlling for the appliance [18].

Further, studies that utilized lip bumper noted that an initial anterior crowding of more than $4 \mathrm{~mm}$ was a risk factor for M2 eruption [15]. When the duration of lip bumper therapy was more than 2 years, the odds of developing M2 eruption disturbance became higher and an altered eruptive path was consequential [15]. Bergersen noted first molar distalization in $95 \%$ of the patients under lip bumper therapy with its increased duration of use coupled with the number of times it was linearly advanced [30]. Another study by Shapira et al. reported that the deficient mesial root length of the M2 as the primary impaction factor [37], but this notion was not analyzed in any of the primary studies in the present systematic review. More research is warranted to extrapolate if the effects on the second molar differ between the appliances (lip bumper/lingual arch).

Studies in the present systematic review measured the outcomes based on their own criteria and had the ages matched between the treatment and control groups [15, $17,18]$. But the definition of the criteria was not consistent across the studies. In one of the included studies, eruption difficulty was defined, when the root of the M2 was at least $75 \%$ formed, but the tooth remained unerupted [17]. In another study, closed apices of the roots irrespective of the $75 \%$ root completion was considered [15]. Another study did not give consideration to root development, instead defined impaction based on cuspal clinical visibility (Table 5) [16]. Another study noted $75 \%$ root completion along with distal cusp clinical visibility [18]. It could be extrapolated that there were no standard outcome measurement criteria employed in the literature.

\section{Limitations}

The limitation of the present systematic review and metaanalysis was the extrapolation of evidence despite the lack of RCTs in this field of research. RCTs and prospective controlled trials are deemed necessary to provide a high-quality evidence. The primary studies that contributed to the review adopted no uniform criteria to measure the clinical outcomes. 
Table 4 Quality assessment using quality assessment tool for observational cohort and cross-sectional studies

\begin{tabular}{|c|c|c|c|c|c|}
\hline Criteria & $\begin{array}{l}\text { Sonis and } \\
\text { Ackerman } \\
{[16]}\end{array}$ & Ferro et al. [15] & Rubin et al. [17] & Jacob et al. [19] & $\begin{array}{l}\text { Arevalo } \\
\text { et al. } \\
{[18]}\end{array}$ \\
\hline Was research question or objective in paper clearly stated? & $\sqrt{ }$ & $\sqrt{ }$ & $\sqrt{ }$ & $\sqrt{ }$ & $\sqrt{ }$ \\
\hline Was study population clearly specified and defined? & $\sqrt{ }$ & $\sqrt{ }$ & $\sqrt{ }$ & $\sqrt{ }$ & $\sqrt{ }$ \\
\hline Was participation rate of eligible persons at least $50 \%$ ? & $\sqrt{ }$ & $\sqrt{ }$ & $\sqrt{ }$ & $\sqrt{ }$ & $\sqrt{ }$ \\
\hline $\begin{array}{l}\text { Were all participants selected or recruited from the same or similar } \\
\text { populations (including the same time period)? Were inclusion } \\
\text { and exclusion criteria for being in study prespecified and applied } \\
\text { uniformly to all participants? }\end{array}$ & $\sqrt{ }$ & $\sqrt{ }$ & $\sqrt{ }$ & $x$ & $\sqrt{ }$ \\
\hline $\begin{array}{l}\text { Was sample size justification, power description, or variance and } \\
\text { effect estimates provided? }\end{array}$ & $x$ & $x$ & $\sqrt{ }$ & $x$ & $\sqrt{ }$ \\
\hline $\begin{array}{l}\text { For analyses in this paper, were the exposure(s) of interest measured } \\
\text { prior to the outcome(s) being measured? }\end{array}$ & $\sqrt{ }$ & $\sqrt{ }$ & $\sqrt{ }$ & $\sqrt{ }$ & $\sqrt{ }$ \\
\hline $\begin{array}{l}\text { Was timeframe sufficient so that one could reasonably expect to see } \\
\text { association between exposure and outcome if it existed? }\end{array}$ & $C D$ & $\sqrt{ }$ & $\sqrt{ }$ & $\sqrt{ }$ & $C D$ \\
\hline $\begin{array}{l}\text { For exposures that can vary in amount or level, did study examine } \\
\text { different levels of exposure as related to outcome (such as catego- } \\
\text { ries of exposure, or exposure measured as continuous variable)? }\end{array}$ & NA & NA & NA & NA & NA \\
\hline $\begin{array}{l}\text { Were exposure measures (independent variables) clearly defined, } \\
\text { valid, reliable, and implemented consistently across all study } \\
\text { participants? }\end{array}$ & $\sqrt{ }$ & $\sqrt{ }$ & $\sqrt{ }$ & $x$ & $\sqrt{ }$ \\
\hline Was exposure(s) assessed more than once over time? & NA & NA & NA & NA & NA \\
\hline $\begin{array}{l}\text { Were outcome measures (dependent variables) clearly defined, valid, } \\
\text { reliable, and implemented consistently across all study partici- } \\
\text { pants? }\end{array}$ & $\sqrt{ }$ & $\sqrt{ }$ & $\sqrt{ }$ & $\sqrt{ }$ & $\sqrt{ }$ \\
\hline $\begin{array}{l}\text { Were outcome assessors blinded to the exposure status of partici- } \\
\text { pants? }\end{array}$ & NA & NA & NA & NA & NA \\
\hline Was loss to follow-up after baseline $20 \%$ or less? & $\sqrt{ }$ & $\sqrt{ }$ & $\sqrt{ }$ & $\sqrt{ }$ & $\sqrt{ }$ \\
\hline $\begin{array}{l}\text { Were key potential confounding variables measured and adjusted } \\
\text { statistically for their impact on relationship between exposure(s) } \\
\text { and outcome(s)? }\end{array}$ & $\sqrt{ }$ & $\sqrt{ }$ & $\sqrt{ }$ & NR & $\sqrt{ }$ \\
\hline Quality rating & Fair & Fair & Good & Poor & Fair \\
\hline
\end{tabular}

$\sqrt{ }$ yes, $x$ no, $C D$ cannot determine, $N A$ not applicable, $N R$ not recorded

\section{Conclusions}

This systematic review demonstrates that ALPS pose a risk for development of mandibular second molar eruption disturbances, but the evidence was of very low quality. Methodologically sound prospective clinical trials are deemed necessary to provide higher levels of evidence.

\section{Implications for practice and future research}

Within the limitations of this systematic review and meta-analysis, the authors intend to highlight a probable impending situation wherein a mandibular anterior discrepancy (crowding) was managed at the expense of creating a posterior discrepancy. The imminent consequence was the development of mandibular second molar eruption disturbances. The key implications are that preserving leeway space increases the risk of impaction of second molars. Further, the chances for development of posterior crowding needs to be considered in the treatment planning process.

Allen et al. reported a secular reduction in the mandibular leeway space in twenty-first century American White population and hypothesized that this reduction could influence the mesial migration of first molars and subsequent M2 eruption [38]. Future research is warranted to study the relationship between secular trends and mandibular leeway space in different races. This would allow for a thorough understanding of the underlying processes in the development of dental arch and help to establish clinical practice guidelines in the use of arch length preservation strategies. Further, with the lack of adequate evidence, the subject matter is a 'hot topic' for the researchers to conduct prospective trials. It is also recommended to perform multicentric studies to reduce the risk of performance bias in eventual RCTs. 


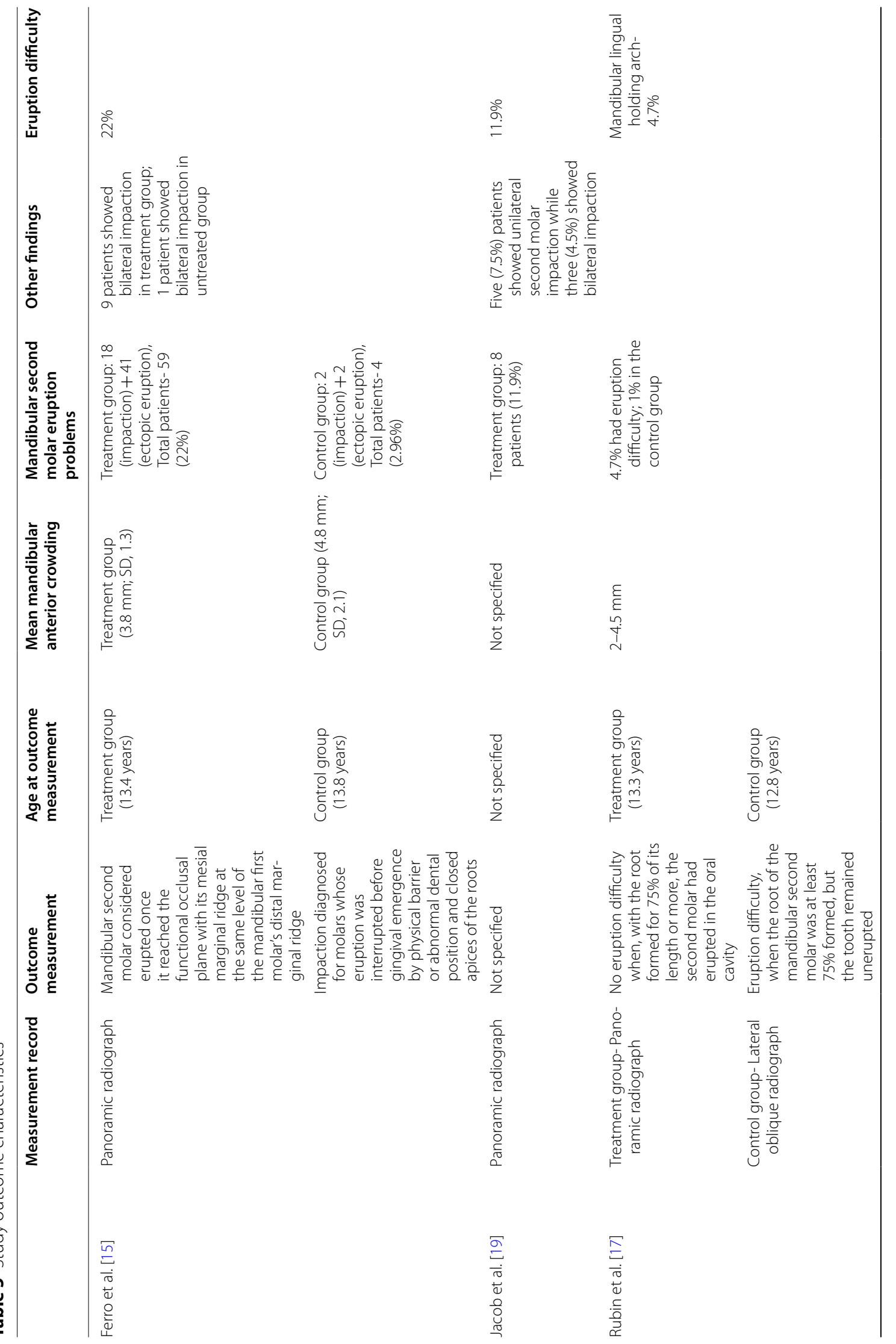




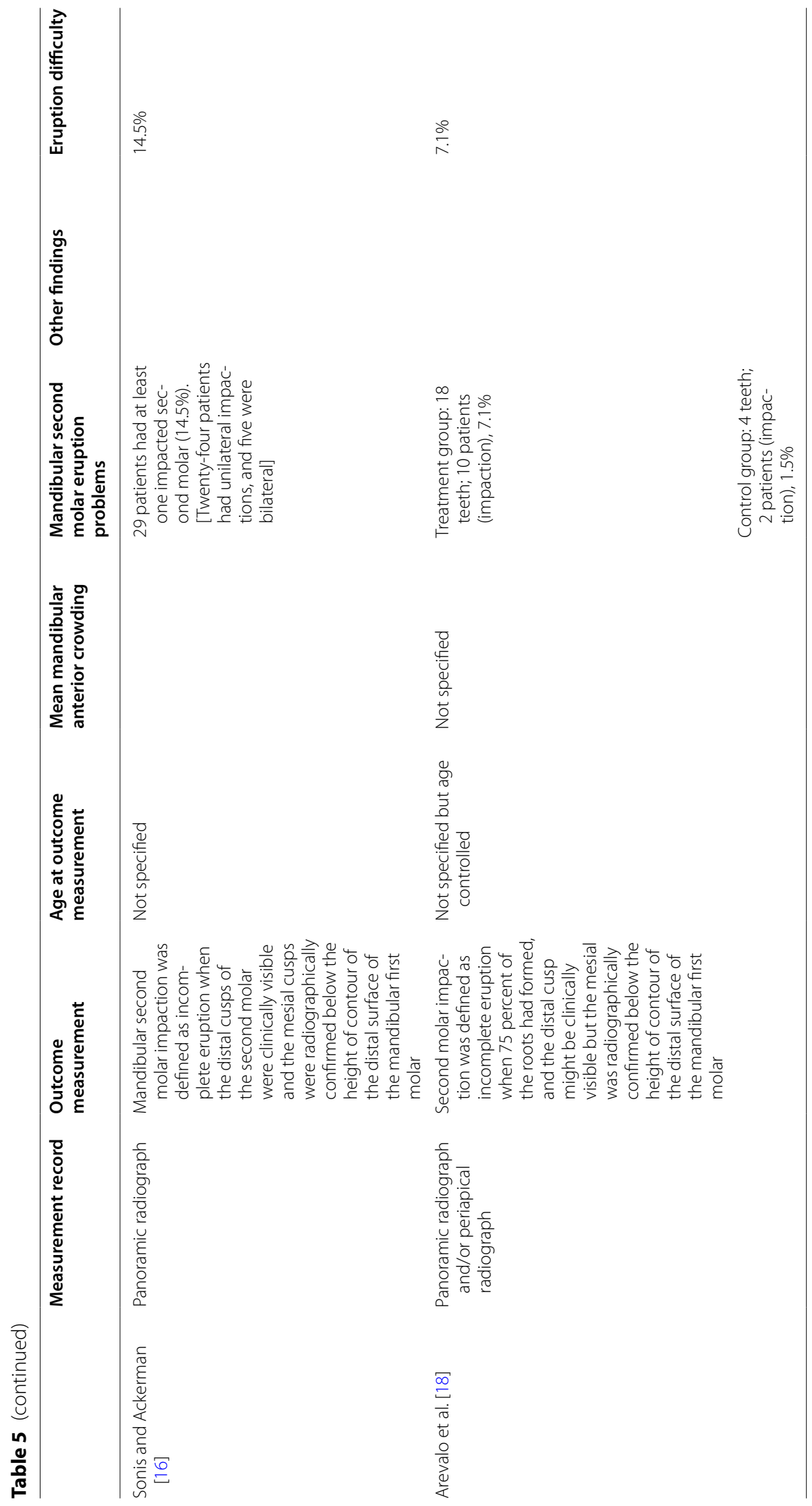




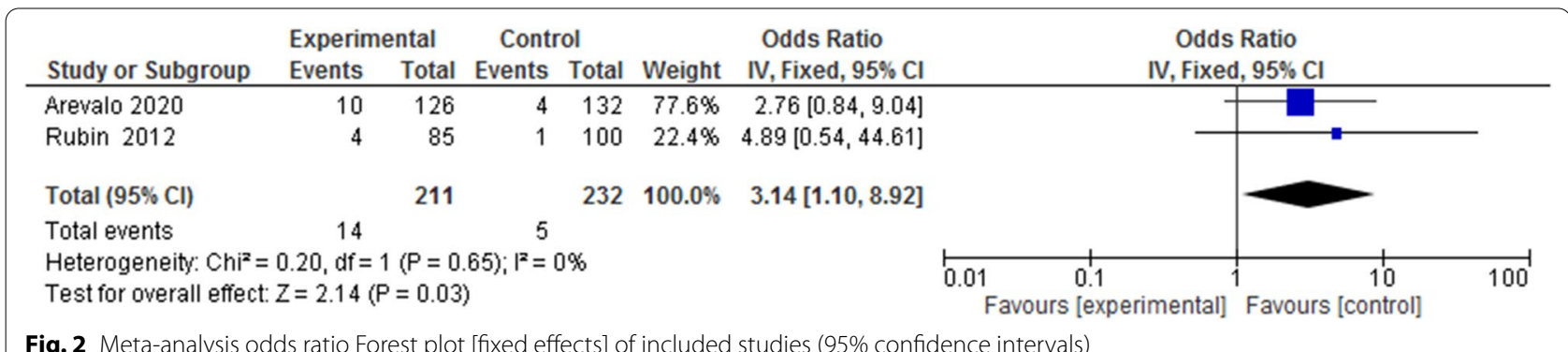

Fig. 2 Meta-analysis odds ratio Forest plot [fixed effects] of included studies (95\% confidence intervals)

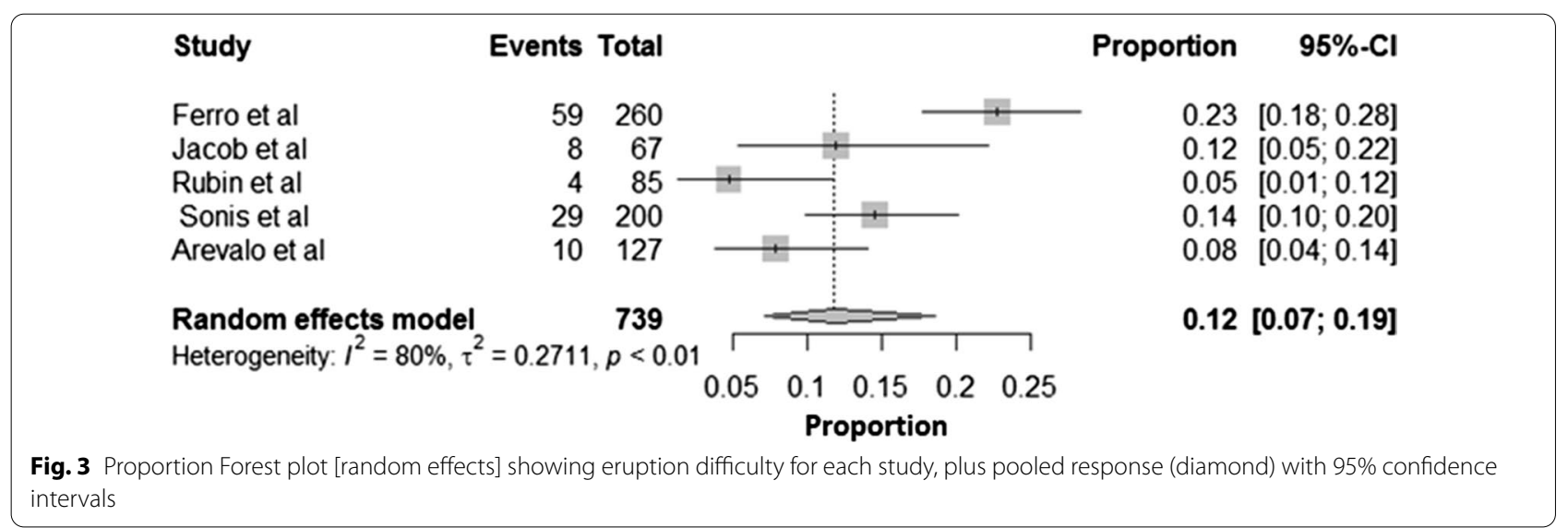

Table 6 GRADE evidence profile [gradepro.org]

\begin{tabular}{|c|c|c|c|c|c|}
\hline \multirow[t]{2}{*}{ Outcomes } & \multirow{2}{*}{$\begin{array}{l}\text { No of participants } \\
\text { (studies) follow up }\end{array}$} & \multirow{2}{*}{$\begin{array}{l}\text { Quality of the evidence } \\
\text { (GRADE) }\end{array}$} & \multirow{2}{*}{$\begin{array}{l}\text { Relative effect } \\
(95 \% \mathrm{CI})\end{array}$} & \multicolumn{2}{|c|}{ Anticipated absolute effects } \\
\hline & & & & Risk with control & $\begin{array}{l}\text { Risk difference with D } \\
(95 \% \mathrm{Cl})\end{array}$ \\
\hline \multirow{4}{*}{$\begin{array}{l}\text { Mandibular second } \\
\text { molar eruption } \\
\text { disturbance }\end{array}$} & 444 (2 studies) & $\oplus \Theta \Theta \Theta$ & $\begin{array}{l}\text { OR } 5.2(1.92 \text { to } \\
14.06)\end{array}$ & Study population & \\
\hline & & VERY LOW'a due to imprecision & & 22 per 1000 & $\begin{array}{l}81 \text { more per } 1000 \text { (from } 19 \\
\text { to } 215 \text { more) }\end{array}$ \\
\hline & & & & Moderate* & \\
\hline & & & & 20 per 1000 & $\begin{array}{l}76 \text { more per } 1000 \text { (from } 18 \\
\text { to } 203 \text { more) }\end{array}$ \\
\hline
\end{tabular}

GRADE Working Group grades of evidence

High quality: Further research is very unlikely to change our confidence in the estimate of effect

Moderate quality: Further research is likely to have an important impact on our confidence in the estimate of effect and may change the estimate

Low quality: Further research is very likely to have an important impact on our confidence in the estimate of effect and is likely to change the estimate

Very low quality: We are very uncertain about the estimate

$\mathrm{Cl}$ confidence interval, $O R$ odds ratio

${ }^{\text {a }}$ Indirectness: the $95 \% \mathrm{Cl}$ is too wide so, downgraded by one level

*The basis for the assumed risk (e.g. the median control group risk across studies) is provided in footnotes. The corresponding risk (and its $95 \%$ confidence interval) is based on the assumed risk in the comparison group and the relative effect of the intervention (and its $95 \% \mathrm{Cl}$ ) 


\begin{abstract}
Abbreviations
ALPS: Arch length preservation strategies; M2: Mandibular second permanent molar; PRISMA: Preferred reporting items for systematic reviews and meta-analyses; PROSPERO: International prospective register of systematic reviews; PECOS: Population, exposure, comparison, outcome, study design; RCTs: Randomized controlled trials; MEDLINE: Medical literature analysis and retrieval system online; ROB: Risk of bias; GRADE: Grading of recommendations assessment, development, and evaluation; Cl: Confidence interval.
\end{abstract}

\section{Acknowledgements}

Not applicable.

\section{Authors' contributions}

SA: Literature search, designing and conceptualization of the review, data collection, data analysis, interpreting data, preparing the manuscript; IS: Data analysis, interpreting data, preparing and revising the manuscript; JJ: Revising the manuscript; IS: Interpreting data and revising the manuscript; All authors read and approved the final manuscript.

\section{Funding}

The systematic review was funded by International Medical University (internal grant). Internal funding (Corresponding author's institution supported the conduct of the research).

\section{Availability of data and materials}

Date and materials are available on request from the corresponding author.

\section{Declarations}

\section{Ethics approval and consent to participate}

The proposal has been approved by IMU Joint-Committee on Research and Ethics (International Medical University, Kuala Lumpur, Malaysia); Project ID: IMU 416/2018; Approval date: 12 November 2018.

\section{Consent for publication}

Not applicable.

\section{Competing interests}

The authors declare no competing interests.

\section{Author details}

${ }^{1}$ School of Dentistry, International Medical University, No.126, Jalan Jalil Perkasa 19, Bukit Jalii, Kuala Lumpur, Malaysia. ${ }^{2}$ Faculty of Dentistry, SEGi University, Kuala Lumpur, Malaysia. ${ }^{3}$ Department of Developmental Dentistry, University of Texas Health School of Dentistry, 7703 Floyd Curl Drive, San Antonio, TX 78229, USA. ${ }^{4}$ Department of Dentistry, AllMS, Bhubaneswar, India.

Received: 17 January 2021 Accepted: 26 July 2021

Published online: 11 August 2021

\section{References}

1. Moorrees CFA, Chada JM. Available space for incisors during dental development. A growth study based on physiologic age. Angle Orthod. 1965;35:12-22.

2. Nance HN. The limitations of orthodontic treatment. I. Mixed dentition diagnosis and treatment. Am J Orthod. 1947;33:177-223.

3. Viglianisi A. Effects of lingual arch used as space maintainer on mandibular arch dimension: a systematic review. Am J Orthod Dentofacial Orthop. 2010;138:382.e1-382.e4.

4. Singer J. The effect of the passive lingual arch on the lower denture Angle Orthod. 1974;44:146-55.

5. Rebellato J, Lindauer SJ, Rubenstein LK, Isaacson RJ, Davidovitch M, Vroom K. Lower arch perimeter preservation using the lingual arch. Am J Orthod Dentofac Orthop. 1997;112:449-56.

6. Brennan MM, Gianelly AA. The use of the lingual arch in the mixed dentition to resolve incisor crowding. Am J Orthod Dentofac Orthop. 2000;117:81-5.
7. Dugoni S, Lee JS, Dugoni A. Early mixed dentition treatment: post retention evaluation of stability and relapse. Angle Orthod. 1995;65:311-20.

8. Chen CY, Hsu KC, Marghalani AA, Dhar V, Coll JA. Systematic review and meta-analysis of passive lower lingual arch for resolving mandibular incisor crowding and effects on arch dimension. Pediatr Dent. 2019:41:9-22.

9. Burden D. Oral health-related benefits of orthodontic treatment. Semin Orthod. 2007:13:76-80.

10. Hafez HS, Shaarawy SM, Al-Sakiti AA, Mostafa YA. Dental crowding as a caries risk factor: a systematic review. Am J Orthod Dentofacial Orthop. 2012;142:443-50

11. Bell RA, Sonis A. Space supervision and guidance of eruption in management of lower transitional crowding: a non-extraction approach. Semin Orthod. 2014;20:16-35.

12. Graber L, Vanarsdall R, Vig K. Orthodontics Current Principles and Techniques. 5th ed. Chapter 15;Philadelphia: Elsevier/Mosby, c2012:532.

13. Grossen J, Ingervall B. The effect of a lip bumper on lower dental arch dimensions and tooth positions. Eur J Orthod. 1995;17:129-34.

14. Paulo SV, Betty BM. Retention probability of permanent mandibular M2s (pilot study). Odonto-stomatología. 2014;16:39-44.

15. Ferro F, Funiciello G, Perillo L, Chiodini P. Mandibular lip bumper treatment and M2 eruption disturbances. Am J Orthod Dentofac Orthop. 2011;139:622-7.

16. Sonis A, Ackerman M. E-space preservation. Is there a relationship to mandibular M2 impaction? Angle Orthod. 2011;81:1045-9.

17. Rubin RL, Baccetti T, McNamara JA Jr. Mandibular M2 eruption difficulties related to the maintenance of arch perimeter in the mixed dentition. Am J Orthod Dentofacial Orthop. 2012;141:146-52.

18. Arevalo SS, Choy R, Rich AP, Felemban O, Bagher SM, Loo CY. Relationship of lower lingual arch appliance use and impaction of M2s: a retrospective study. Pediatr Dent. 2020;42:123-5.

19. Jacob HB, LeMert S, Alexander RG, Buschang PH. M2 impaction associated with lip bumper therapy. Dental Press J Orthod. 2014;19:99-104.

20. Bondemark L, Tsiopa J. Prevalence of ectopic eruption, impaction, retention and agenesis of the permanent M2. Angle Orthod. 2007;77:773-8.

21. Magnusson C, Kjellberg H. Impaction and retention of M2s: diagnosis, treatment and outcome. A Retrospective follow-up study. Angle Orthod. 2009;79:422-7.

22. Moher D, Shamseer $L$, Clarke M, Ghersi D, Liberati A, Petticrew M, Shekelle P, Stewart LA, PRISMA-P Group. Preferred reporting items for systematic review and meta-analysis protocols (PRISMA-P) 2015 statement. Syst Rev. 2015:4:1.

23. National Institutes of Health (2014). U.S. Department of Health \& Human Services. Quality Assessment Tool for Observational Cohort and CrossSectional Studies. Available at: http://www.nhlbi.nih.gov/health-pro/ guidelines/in-develop/cardiovascular-risk-reduction/tools/cohort.htm. Accessed, December, 2019

24. Landis JR, Koch GG. The measurement of observer agreement for categorical data. Biometrics. 1977;33:159-74.

25. Higgins JPT, Thomas J, Chandler J, Cumpston M, Li T, Page MJ, Welch VA (editors). Cochrane Handbook for Systematic Reviews of Interventions version 6.0 (updated July 2019). Cochrane, 2019. Available at: www. training.cochrane.org/handbook.

26. Higgins JP, Thompson SG, Deeks JJ, Altman DG. Measuring inconsistency in meta-analyses. BMJ. 2003;327(7414):557-60.

27. Di Luzio C, Bellisario A, Giovannoni D, Caputo M. M2 impaction with lip bumper therapy. Webmed Central Orthod. 2017;8:WMC005410.

28. Santana LG, de Campos FE, Flores-Mir C, Abreu LG, Marques LS, MartinsJunior PA. Effects of lip bumper therapy on the mandibular arch dimensions of children and adolescents: a systematic review. Am J Orthod Dentofac Orthop. 2020;157:454-465.e1.

29. Davidovitch M, Mclnnis D, Lindauer SJ. The effects of lip bumper therapy in the mixed dentition. Am J Orthod Dentofac Orthop. 1997;111:52-8.

30. Bergersen EO. A cephalometric study of the clinical use of the mandibular labial bumper. Am J Orthod. 1972;61:578-602.

31. Werner SP, Shivapuja PK, Harris EF. Skeletodental changes in the adolescent accruing from use of the lip bumper. Angle Orthod. 1994:64:13-22.

32. Nevant CT, Buschang PH, Alexander RG, Steffen JM. Lip bumper therapy for gaining arch length. Am J Orthod Dentofac Orthop. 1991;100:330-6.

33. Raucci G, Pachêco-Pereira C, Elyasi M, d'Apuzzo F, Flores-Mir C, Perillo L. Short- and long-term evaluation of mandibular dental arch dimensional 
changes in patients treated with a lip bumper during mixed dentition followed by fixed appliances. Angle Orthod. 2016;86:753-60.

34. O'Neill J. Do lip bumpers work? Evid Based Dent. 2009;10:48-9.

35. Osborn WS, Nanda RS, Currier GF. Mandibular arch perimeter changes with lip bumper treatment. Am J Orthod Dentofacial Orthop. 1991;99(6):527-32.

36. Saatci P, Ustun P, Vural G. Effects of lip bumper therapy on unerupted second molars. Eur J Orthod. 1999;5:618.

37. Shapira Y, Finkelstein T, Shpack N, Lai YH, Kuftinec MM, Vardimon A. Mandibular M2 impaction. Part I: Genetic traits and characteristics. Am J Orthod Dentofac Orthop. 2011;140:32-7.
38. Allen TR, Trojan TM, Harris EF. Evidence favoring a secular reduction in mandibular leeway space. Angle Orthod. 2017;87:576-82.

\section{Publisher's Note}

Springer Nature remains neutral with regard to jurisdictional claims in published maps and institutional affiliations.
Ready to submit your research? Choose BMC and benefit from:

- fast, convenient online submission

- thorough peer review by experienced researchers in your field

- rapid publication on acceptance

- support for research data, including large and complex data types

- gold Open Access which fosters wider collaboration and increased citations

- maximum visibility for your research: over $100 \mathrm{M}$ website views per year

At BMC, research is always in progress.

Learn more biomedcentral.com/submissions 